\title{
Spatiotemporal Expression and Substrate Specificity Analysis of the Cucumber SWEET Gene Family
}

\author{
Yaxin $\mathrm{Li}^{\dagger}$, Sheng Feng', Si Ma, Xiaolei Sui* and Zhenxian Zhang* \\ Beijing Key Laboratory of Growth and Developmental Regulation for Protected Vegetable Crops, College of Horticulture, \\ China Agricultural University, Beijing, China
}

\section{OPEN ACCESS}

Edited by:

Bernard Grodzinski,

University of Guelph, Canada

Reviewed by:

Cankui Zhang

Purdue University, United States

Alina Tollenaere,

The University of Queensland,

Australia

${ }^{*}$ Correspondence:

Zhenxian Zhang

zhangzx@cau.edu.cn

Xiaolei Sui

sui-office@cau.edu.cn

tThese authors have contributed equally to this work.

Specialty section: This article was submitted to Plant Breeding,

a section of the journal Frontiers in Plant Science

Received: 05 June 2017 Accepted: 11 October 2017 Published: 27 October 2017

Citation:

Li Y, Feng S, Ma S, Sui X and Zhang Z (2017) Spatiotemporal Expression and Substrate Specificity

Analysis of the Cucumber SWEET Gene Family. Front. Plant Sci. 8:1855.

doi: 10.3389/fpls.2017.01855
The functions of SWEET (Sugar Will Eventually be Exported Transporter) proteins have been studied in a number of crops, but little is known about their roles in cucumber (Cucumis sativus L.), a model plant for studying stachyose metabolism and phloem function. Here, we identified 17 cucumber SWEET genes (CSSWEETS), located on chromosomes 1-6, and classified them into four clades. Two genes from each clade were selected for spatiotemporal expression, subcellular localization, and substrate specificity analyses. Clade I and II proteins were all hexose transporters and targeted to the plasma membrane, while clade III proteins also localized to the plasma membrane, but used sucrose as a substrate. Clade IV SWEET proteins were localized to the tonoplast, and used hexose as a substrate. The eight tested CSSWEET genes were most highly expressed in flower, which represents a large sink in plants. However, each gene also showed specific expression patterns: three of the eight tested genes were highly expressed in mature leaves, two in roots, two in fruit, two in stems, and one was detected in all tested organs. The likely biological roles of each are discussed based on the above results.

Keywords: cucumber, phylogeny, spatiotemporal expression, subcellular localization, substrate specificity, sugar, SWEET transporters

\section{INTRODUCTION}

Sugar Will Eventually be Exported Transporters (SWEET) proteins are membrane localized proteins with seven transmembrane domains (TMs) that have been identified as sugar transporters (STPs) by co-expression studies with the high-sensitivity fluorescence resonance energy transfer (FRET) glucose/sucrose sensor in human HEK293T cells (Chen et al., 2010; Xuan et al., 2013; Eom et al., 2015). Unlike sucrose transporter (SUT), STP (symporter) or vacuolar glucose transporter 1 (VGT1) (antiporter), SWEET proteins are energy-independent and their action is driven by a substrate concentration gradient across the membrane (Chen et al., 2015a). SWEET proteins are widespread in land plants, but have also been found in algae (Chlamydomonas reinhardtii), nematodes (Caenorhabditis elegans), and in mammals (Chen et al., 2010). In angiosperms, the number of reported SWEET genes varies from 8 to 52, depending on the species, with 8 in the earliest diverging angiosperm, Amborella trichopoda, 17 in Arabidopsis thaliana, 17 in grapevine (Vitis vinifera), 21 in rice (Oryza sativa), 23 in sorghum (Sorghum bicolor), 29 in tomato (Solanum lycopersicum), 35 in potato (S. tuberosum), 47 in eucalyptus (Eucalyptus grandis), and 52 in soybean 
(Glycine max) (Chen et al., 2010; Eom et al., 2015; Feng et al., 2015; Manck-Götzenberger and Requena, 2016; Mizuno et al., 2016).

Chen et al. (2010) first classified SWEET transporters into four clades, and identified differences in their subcellular localization, substrates and functions. SWEET proteins from clades I and II preferentially transport hexoses, mainly glucose, and they have been reported to be localized to the plasma membrane, with the exception of SWEET2 from A. thaliana. AtSWEET5 (VEX1) is expressed in mature, hydrated and germinating pollen and is found specifically in the vegetative cell of pollen grains, consistent with a role in supplying the generative cell with sugars (Engel, 2005; Eom et al., 2015). In rice, the clade II protein, OsSWEET5, is a galactose transporter and is expressed in anthers. OsSWEET5overexpressing plants were shown to display a growth retardation phenotype and precocious senescence at the seedling stage (Zhou et al., 2014). The clade II protein AtSWEET8 (RPG1) is preferentially expressed in inflorescences, and may transport sugar to the tapetum and the microsporocyte/microspores, which function as a subunit for the synthesis of primexine precursors and callose during the meiosis and tetrad stage. Knocking out the expression of AtSWEET8 resulted in reduced primexine deposition and pollen degradation (Guan et al., 2008; Sun et al., 2013). ZmSWEET4c and OsSWEET4 also appeared to be responsible for transferring hexoses across the basal endosperm transfer layer (BETL) to sustain development of the large starchstoring endosperm of cereal grains and contribute to sink strength (Sosso et al., 2015).

SWEET proteins from clade III are sucrose transporters and are targeted to the plasma membrane (Chen et al., 2012). Genes in this clade have various functions, including exporting sucrose from the phloem parenchyma cells (PPC) prior to phloem loading in source tissues, embryo development, nectar secretion and pollen nutrition by the tapetum, which are mostly symplastisolation spaces in sink tissues (Chen et al., 2012, 2015b; Sun et al., 2013; Lin et al., 2014). SWEET9, also referred to NEC1 in petunia (Wittich, 2000), has been identified as being nectar-specific and to play essential roles in nectar secretion in A. thaliana, Brassica rapa, and Nicotiana attenuata. Specifically, the protein acts to release sucrose from the nectary parenchyma cells to the apoplast, whereupon it is hydrolyzed by cell wall invertases to produce glucose and fructose (Lin et al., 2014). AtSWEET11 and -12 , also from clade III, release sucrose from leaf PPCs to the apoplast, after which it is trafficked by SUT transporters into the sieve element-companion cell complex (Chen et al., 2012; Eom et al., 2015). The atsweet 11;12 double mutant was reported to accumulate starch in its leaves and the cumulative exudation of $\left[{ }^{14} \mathrm{C}\right]$-derived assimilates from ${ }^{14} \mathrm{CO}_{2}$ fed cut petioles was reduced (Chen et al., 2012), providing strong evidence for the corresponding proteins functioning in phloem loading. The rice ortholog, OsSWEET11 (Os8N3/Xa13), is also shown to be a sucrose transporter (Chen et al., 2012). In a later study, it was found that AtSWEET proteins also contribute to assimilate transfer from the maternal seed coat to the developing embryo (Chen et al., 2015b). AtSWEET11, -12, and -15 are expressed in the seed coat and endosperm, and the corresponding triple knockout mutant showed a severe delay in embryo development, as well as a wrinkled seed phenotype at maturity due to a low starch and lipid content and a smaller embryo (Chen et al., 2015b; Eom et al., 2015). AtSWEET15 (SAG29) functions in remobilization of carbohydrates during senescence (Seo et al., 2011), and AtSWEET13 (RPG2) is shown to partially alleviate the loss of AtSWEET8 function at the late reproductive stage in the atsweet 8 mutant, suggesting some level of redundancy. In addition, the atsweet8;13 double mutant shows a high degree of sterility at the late reproductive stage, with small siliques and few seeds (Sun et al., 2013).

SWEET proteins from Clade IV are vacuolar hexose transporters, and may play roles in balancing intracellular hexose homeostasis. As an example, AtSWEET17 functions as a fructosespecific uniporter in the root and leaf tonoplast, thereby playing a key role in facilitating bi-directional fructose transport across the tonoplast of the roots and leaves to maintain cytosolic fructose homeostasis (Chardon et al., 2013; Guo et al., 2014).

Additionally, SWEET protein function has been associated with pathogen infection and it was reported that the expression of various SWEET genes can be induced by biotrophic bacteria or fungi, to release sugar to the apoplast, thereby promoting pathogen growth (Chen et al., 2010; Chen, 2014). This phenomenon has been confirmed in many crop plants (Yuan et al., 2009; Antony et al., 2010; Chen et al., 2010; Liu et al., 2011; Chen, 2014; Chong et al., 2014; Manck-Götzenberger and Requena, 2016).

Although considerable progress has been made in the study of SWEET proteins from many plant species, to date little is known about their expression or function in a cucurbit, even though members of the Cucurbitaceae are model plants for studying stachyose metabolism, as well as phloem function. Recently, the cucumber (Cucumis sativus L.) genome sequence was published (Huang et al., 2009), providing a basis for studying the cucumber SWEET gene family. Hu et al. (2017) identified 17 SWEET transporters in cucumber by bioinformatic analysis. In this current study, phylogenetic analyses, yeast uptake assays, quantitative real-time PCR (qRT-PCR), and green fluorescent protein (GFP) fusion protein localization were used to determine the classification, substrate specificity, spatiotemporal expression profiles, and subcellular localization of the cucumber SWEET genes/proteins. The resulting data are used to infer their likely biological functions, thereby providing a theoretical basis for further research of SWEET proteins in cucurbit species.

\section{MATERIALS AND METHODS}

\section{Plant Material and Bacteria/Yeast Strains}

Cucumber (C. sativus L. cv. Xintaimici) plants were grown under glasshouse conditions with $28 / 18^{\circ} \mathrm{C}$ (day/night) temperature and 12/12 h (light/dark) photoperiod in Beijing, China. Tissue including root, stem, young leaf (sink leaf in apical growing point), mature leaf (functional leaf), flowers, and fruit were collected from 3-month-old plants and used for qRT-PCR analyses. The DH5a Escherichia coli strain was used for cloning and Saccharomyces cerevisiae strains EBY.VW4000 and SUSY7/ura were used for heterologous protein expression. 


\section{Identifying Members of the Cucumber SWEET Gene Family and Phylogenetic Analyses}

CsSWEET genes were identified via BLASTN searches in the National Center for Biotechnology Information $\left(\mathrm{NCBI}^{1}\right)$ database and the Cucurbit Genomics Database $\left(\mathrm{CuGenDB}^{2}\right)$. The resulting 17 cucumber putative SWEET genes were named CsSWEET1-17, with the numbering based on their homologous genes in A. thaliana (Chen et al., 2010). The letter corresponds to the location on chromosome and Hu et al. (2017).

Phylogenetic analyses were conducted using ClustalW in Mega 5.0 software and an unrooted phylogenetic tree of the CsSWEET, AtSWEET, and SISWEET families were constructed using Mega 5.0 software according to Chen et al. (2010). The evolutionary history was inferred using the neighbor joining method with 1,000 replicates. The evolutionary distances were computed using the Poisson correction distance model and are given as the number of amino acid substitutions per site.

\section{Gene Structure and Transmembrane Domain Analysis}

Information on the SWEET genes was acquired from NCBI, including locus tags, gene symbols, accession numbers, the chromosomal position and the MtN3 saliva family (PFAM database code $\mathrm{PF} 03083^{3}$ ) analysis. Structure of each CsSWEET gene was analyzed using NCBI. The TMs were analyzed using $\mathrm{TMHMM}^{4}$.

\footnotetext{
${ }^{1}$ http://www.ncbi.nlm.nih.gov/

${ }^{2}$ http://www.icugi.org/cgi-bin/ICuGI/index.cgi

${ }^{3}$ http://pfam.xfam.org

${ }^{4} \mathrm{http}: / /$ www.cbs.dtu.dk/services/TMHMM
}

\section{Heterologous Expression of CsSWEET in Yeast}

The CsSWEET1, CsSWEET2, CsSWEET5a, CsSWEET7b, CsSWEET10, CsSWEET12c, CsSWEET17a, and CsSWEET17c open reading frames (ORFs) were amplified by PCR and cloned into the $S$. cerevisiae/E. coli shuttle vector pDR196 (Fan and Zhang, 2009). PCR primers listed in Supplementary Table S3.

The recombinant vectors or the empty pDR196 vector (control) were separately transferred into the hexose uptakedeficient yeast strain EBY.VW4000 and sucrose uptake-deficient yeast strain SUSY7/ura, as previously described (Cheng et al., 2015). The transformed cells of the hexose uptake-deficient strain were grown on SD (synthetic deficient) medium supplemented with $2 \%$ maltose (glucose for sucrose uptake-deficient strain) and auxotrophic requirements. Serial dilutions $\left(\times 10, \times 10^{2}, \times 10^{3}\right)$ of yeast cell suspensions (CsSWEET1, CsSWEET2, CsSWEET5a, CsSWEET7b, CsSWEET17a, and CsSWEET17c) were dropped on solid SD media containing either $2 \%$ maltose (control) or $2 \%$ glucose/fructose/galactose/mannose/xylose/arabinose plus respective auxotrophic requirements (Cheng et al., 2015). Serial dilutions $\left(\times 10, \times 10^{2}, \times 10^{3}\right)$ of CsSWEET10, CsSWEET12c were plated on SD media containing either $2 \%$ glucose (control) or $2 \%$ sucrose plus respective auxotrophic requirements. Growth was documented by pictures after $2-5$ days growth at $30^{\circ} \mathrm{C}$.

\section{Expression Profiles of Cucumber SWEET Genes}

Gene expression analyses were performed using qRT-PCR analysis with the SYBR green detection protocol (TaKaRa, Japan) and the ABI 7500 system (Bio-Rad, United States). Total RNA was extracted from specified tissues (root, stem, young leaf, mature leaf, male flower, female flower, fruit on the day of

TABLE 1 | Cucucmber SWEETs information.

\begin{tabular}{|c|c|c|c|c|c|c|c|c|c|c|c|}
\hline $\begin{array}{l}\text { Gene name } \\
\text { CsSWEET1 }\end{array}$ & $\begin{array}{l}\text { Locus tag } \\
\text { Csa6M343690.1 }\end{array}$ & $\begin{array}{l}\text { Gene symbol } \\
\text { LOC101217779 }\end{array}$ & \multicolumn{2}{|c|}{ Accession } & $\begin{array}{l}\text { AA } \\
252\end{array}$ & $\begin{array}{c}\text { Chr } \\
6\end{array}$ & $\begin{array}{l}\text { Gene } \\
3856\end{array}$ & $\begin{array}{c}\text { Exon } \\
6\end{array}$ & $\begin{array}{c}\text { TMs } \\
7\end{array}$ & \multicolumn{2}{|c|}{ MtN3 } \\
\hline CsSWEET2 & Csa4M622870.1 & LOC101220605 & XM_004146030.2 & XP_004146078.2 & 233 & 4 & 2787 & 6 & 7 & $16-102$ & $135-218$ \\
\hline CsSWEET5a & Csa1M046010.1 & LOC101222361 & XM_004152504.2 & XP_004152552.1 & 238 & 1 & 1546 & 6 & 7 & $10-95$ & $133-218$ \\
\hline CsSWEET5b & Csa3M836410.1 & LOC101217357 & XM_004141139.2 & XP_004141187.1 & 236 & 3 & 1910 & 6 & 6 & $19-95$ & $131-212$ \\
\hline CsSWEET5c & Csa3M836420.1 & LOC101217589 & XM_011653972.1 & XP_011652274.1 & 247 & 3 & 4626 & 6 & 7 & $9-94$ & $134-214$ \\
\hline CsSWEET7b & Csa5M148830.1 & LOC101206775 & XM_004143811.2 & XP_004143859.1 & 265 & 5 & 2582 & 5 & 7 & $10-98$ & $134-218$ \\
\hline CsSWEET9 & Csa2M083730.1 & LOC101218764 & XM_004138930.2 & XP_004138978.2 & 262 & 2 & 3902 & 6 & 7 & $11-97$ & $134-206$ \\
\hline CsSWEET10 & Csa1M001300.1 & LOC101210005 & XM_004137984.2 & XP_004138032.1 & 292 & 1 & 1876 & 5 & 7 & $10-96$ & $131-211$ \\
\hline CsSWEET12a & Csa1M001290.1 & LOC101221611 & XM_004138202.2 & XP_004138250.1 & 275 & 1 & 1523 & 5 & 7 & $13-100$ & $135-219$ \\
\hline CsSWEET12b & Csa1M051710.1 & LOC101212917 & XM_004153453.1 & XP_004153501.1 & 295 & 1 & 2238 & 6 & 7 & $13-99$ & $134-219$ \\
\hline CsSWEET12c & Csa1M051720.1 & LOC101221861 & XM_004145098.2 & XP_004145146.2 & 291 & 1 & 1824 & 6 & 7 & $12-98$ & $133-218$ \\
\hline CsSWEET17c & Csa3M159450.1 & LOC101206107 & XM_004134172.2 & XP_004134220.1 & 244 & 3 & 3820 & 6 & 7 & $6-91$ & $129-213$ \\
\hline
\end{tabular}

Data in this table are from NCBI database. AA, amino acid, Chr, chromosome, TMs, transmembrane domains, MtN3, MtN3 saliva family (PFAM database code PF03083; http://pfam.xfam.org). 


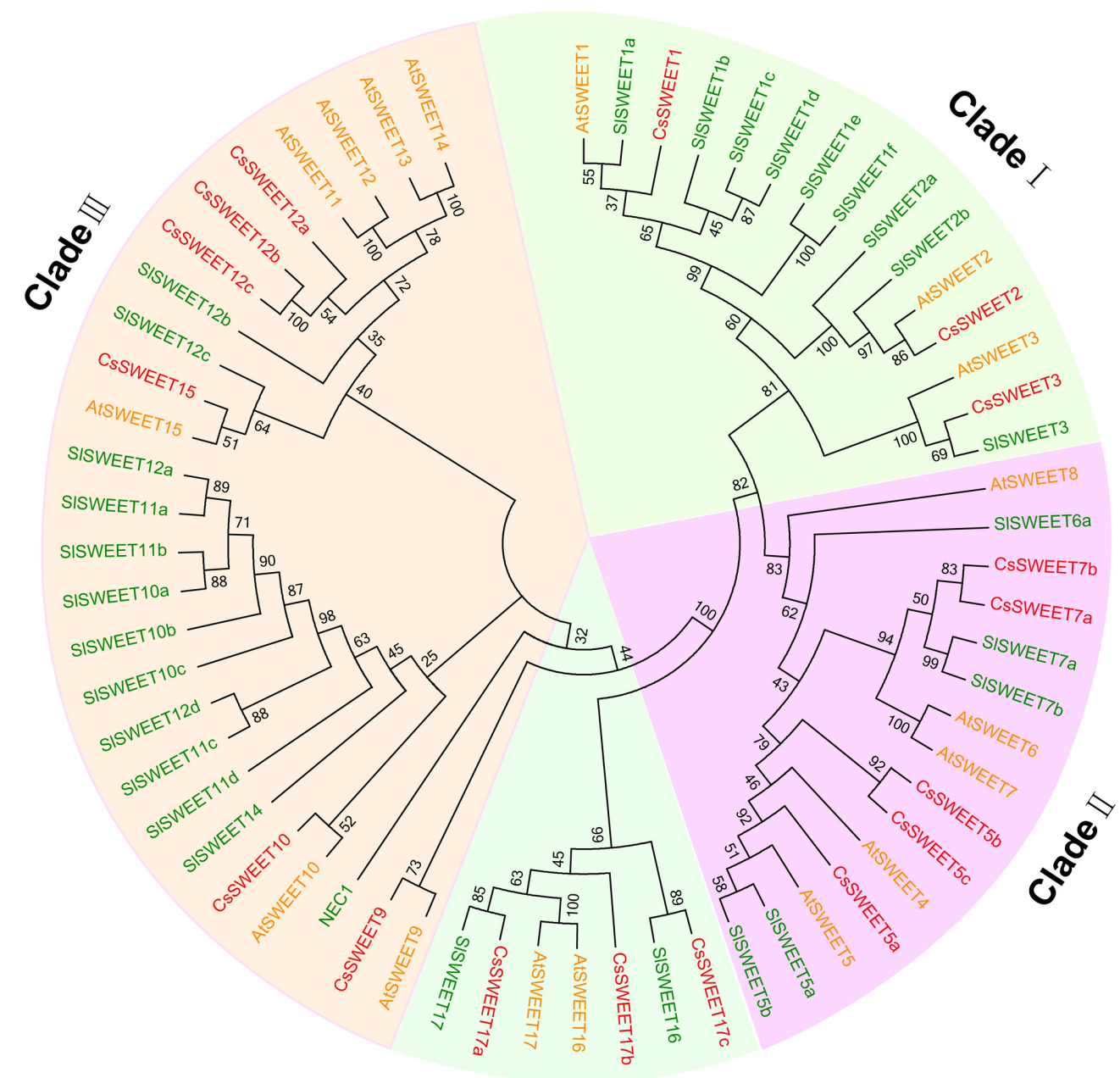

Clade IV

FIGURE 1 | Phylogenetic tree of cucumber (Cucumis sativus), Arabidopsis thaliana, and tomato (Solanum lycopersicum) SWEET proteins. AtSWEET is A. thaliana (yellow), CsSWEET is Cucumis sativus (red), and SISWEET is Solanum lycopersicum (green). The evolutionary history was inferred using the neighbor joining method with 1,000 replicates. The evolutionary distances were computed using the Poisson correction distance model and are in the units of the number of amino acid substitutions per site. Evolutionary analyses were conducted using MEGA5.0 software. Accessions are listed in Supplementary Table S2.

anthesis and fruit on the ninth day after anthesis) using RNAprep pure Plant Kit (Tiangen, Beijing, China), and then reversetranscribed using FastQuant RT Kit (with gDNase) (Tiangen, Beijing, China). The cDNA samples were then used as a template for qRT-PCR analysis. Primers are listed in Supplementary Table S3.

\section{Subcellular Localization of CsSWEET Proteins}

The CsSWEET1, CsSWEET7b, CsSWEET12c, and CsSWEET17a ORFs without stop codons were amplified by RT-PCR using gene specific primers and the amplicons were cloned into the pCAMBIA super 1300 vector to generate C-terminal fusions with the GFP reporter. Primers are listed in Supplementary Table S3. Transient expression of the CsSWEET1-GFP, CsSWEET7b-GFP, CsSWEET12c-GFP, and CsSWEET17a-GFP fusion proteins in
A. thaliana protoplasts and onion epidermal cells were performed according to Ma et al. (2008) and Fan and Zhang (2009), respectively. The empty vector expressing untargeted GFP was used as a control. GFP fluorescence was visualized using an Olympus Confocal Laser Scanning Microscope (FV1000, Japan).

\section{RESULTS}

\section{Identification and Phylogenetic Analysis of the Cucumber SWEET Family}

Seventeen candidate cucumber SWEET genes (CsSWEET) were identified and named according to their homologs in A. thaliana (Chen et al., 2010) (Table 1). Sequences of the CsSWEETs were based on BLAST searches against the NCBI $\left(\mathrm{NCBI}^{5}\right)$ database

\footnotetext{
${ }^{5}$ http://www.ncbi.nlm.nih.gov/
} 


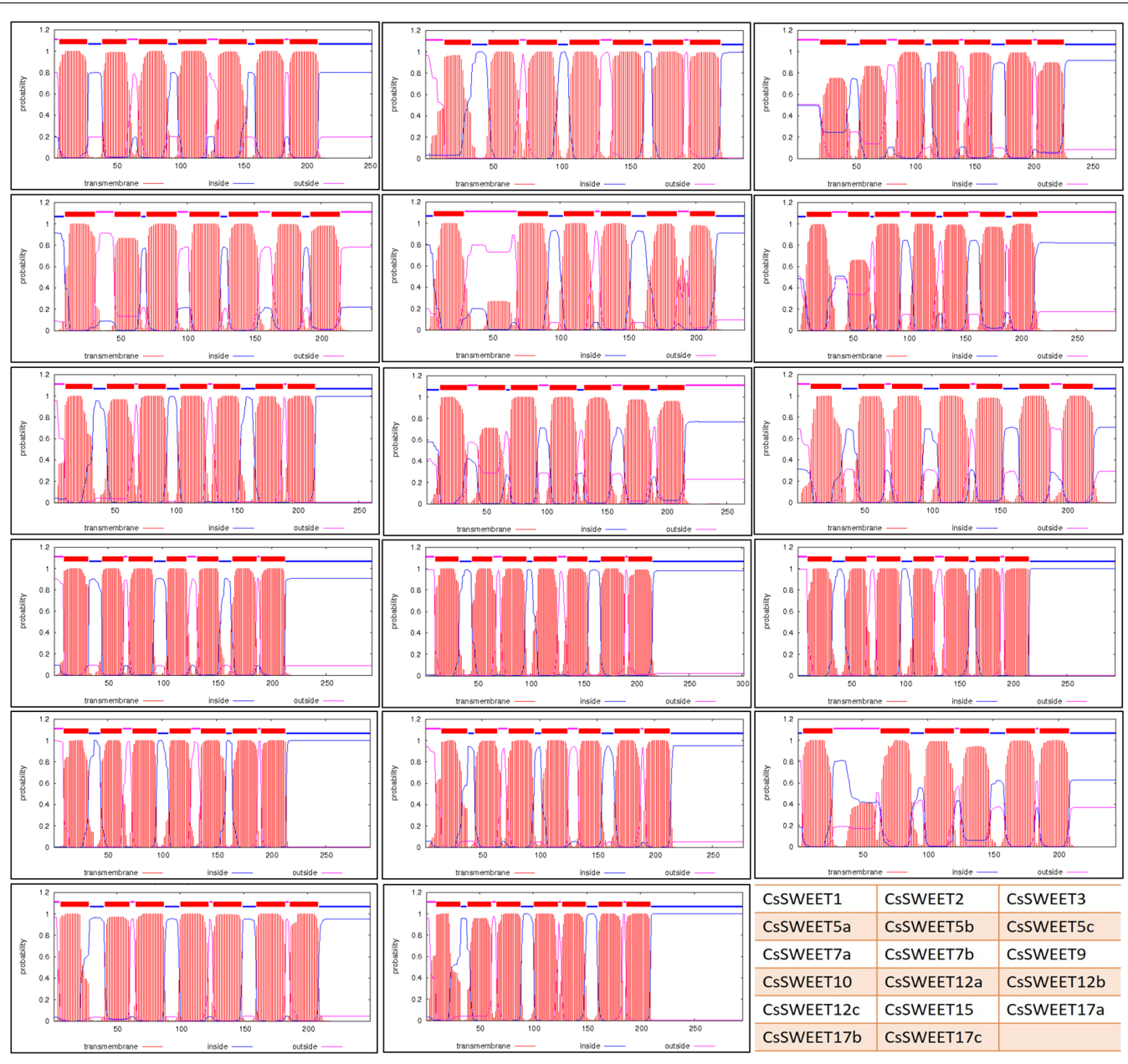

FIGURE 2 | The transmembrance domains of CSSWEET proteins. The position of $\mathrm{N}$ - and C-terminal domains of the protein are indicated by blue or pink lines. Table on the right bottom shows the location of each protein in this figure. The website used for predictions was TMHMM.

and Cucurbit Genomics Database $\left(\mathrm{CuGenDB}^{6}\right)$. A phylogenetic tree based on the 17 CsSWEET protein sequences, together with 17 AtSWEET sequences and 29 SISWEET sequences was constructed using MEGA5.0 software. The cucumber sequences clustered into the four clades that have been reported in the A. thaliana and O. sativa families (Chen et al., 2010) (Figure 1). Clade I contained CsSWEET1-3, besides, three AtSWEETs and nine SISWEETs; Clade II contained CsSWEET5a-c, 7a$\mathrm{b}$, besides, five AtSWEETs and five SISWEETs; Clade III contained CsSWEET9-10, 12a-c, 15, besides, seven AtSWEETs and thirteen SISWEETs; Clade IV contained CsSWEET17a-c, besides, two AtSWEETs and two SISWEETs. Sequence identity comparisons among the four CsSWEET protein subclasses were $28-77 \%$, and the average identity was $42 \%$. CsSWEET $12 \mathrm{~b}$ and CsSWEET12c showed the highest homology (77\%), CsSWEET5b and CsSWEET5c, CsSWEET7a, and CsSWEET7b had 64 and 62\% homology, respectively (Supplementary Table S1).

${ }^{6}$ http://cucurbitgenomics.org/

\section{Gene Structure and Transmembrane Domain Analysis}

To analyze the structural characteristics of the CSSWEET genes, we examined their conserved regions, and aligned the putative protein sequences. Their exon numbers varied among the genes, CsSWEET7a, 7b, CsSWEET10, and CsSWEET12a containing five exons, and the other gene family members all containing six exons (Table 1).

SWEET proteins have seven TMs in eukaryotes, as well as two MtN3/saliva domains. They contain a basic 3-TM unit and a functional transporter containing at least four TMs (tetramer) (Xuan et al., 2013). There are 80-90 amino acids in the two cucumber MtN3/saliva domains, and they are present at almost the same positions in all the proteins, as shown in Table 1.

To confirm the presence of the TMs, the amino acid sequences were submitted to the TMHMM Server v.2.0 ${ }^{7}$. The results confirmed that all, except 2 of the 17 CsSWEETs have seven TMs, CsSWEET5b and CsSWEET17a, which have six

${ }^{7}$ http://www.cbs.dtu.dk/services/TMHMM 


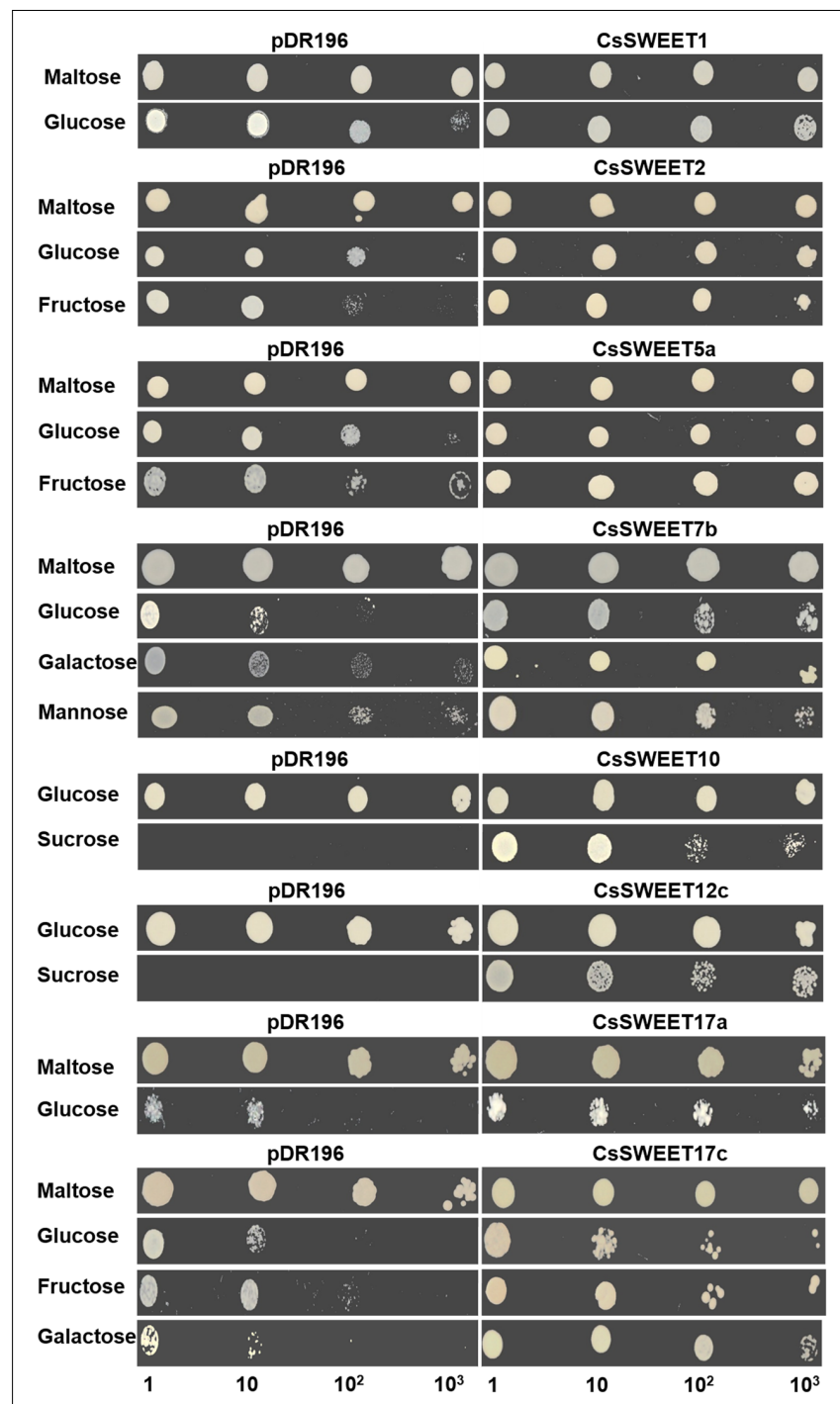

FIGURE 3 | Heterologous expression of eight CSSWEET genes in yeast. Yeast strains with recombinant vectors or the empty pDR196 vector (as a negative control) were grown on SD (synthetic deficient)-ura medium supplemented with $2 \%$ maltose or different sugars (listed above) as the sole carbon source for 5 days. Medium with maltose as sole carbon source is a positive control for strain EBY.WV4000, while with glucose as sole carbon source is a positive control for strain SUSY7/ura. Yeast cell suspensions were diluted $\left(\times 10, \times 10^{2}, \times 10^{3}\right)$ for serial dilutions assay. The other results of the experiment are in Supplementary Figure S2.

TMs (Figure 2). The N-terminal of CsSWEET1, 2, 3, 7a, 9, $10,12 \mathrm{a}, 12 \mathrm{~b}, 12 \mathrm{c}, 15,17 \mathrm{~b}, 17 \mathrm{c}$ is outside of the membrane, while it is on the inside for CsSWEET5a, 5b, 5c, 7b, 17a (Figure 2).

\section{Heterologous Expression and Substrate Specificity Analysis in Yeast}

In order to identify the substrates being transported by the CsSWEET proteins, the hexose uptake-deficient yeast mutant EBY.VW4000 and the sucrose uptake-deficient yeast mutant
SUSY7/ura3 (Riesmeier et al., 1992; Wieczorke et al., 1999) were used. We isolated six full-length cDNA clones, which encoded putative hexose transporters and two full-length cDNA clones, which encoded putative sucrose transporters, from cucumber male flowers. We then determined that CsSWEET1 and CsSWEET2 from clade I allowed glucose uptake, or glucose and fructose uptake, in the yeast EBY.VW4000 mutant (Figure 3), but did not show detectable complementation of uptake of other hexoses (Supplementary Figure S2). From clade II, CsSWEET5a supported uptake of glucose and fructose, while CsSWEET7b conferred glucose, galactose and mannose uptake (Figure 3), but not of other hexoses. From clade III, both CsSWEET10 and CsSWEET12c conferred sucrose uptake in the yeast SUSY7/ura3 mutant but did not allow hexose uptake in the EBY.VW4000 mutant (Figure 3 and Supplementary Figure S2). Finally, from clade IV, CsSWEET17a allowed glucose uptake in the yeast EBY.VW4000 mutant, while CsSWEET17c enabled glucose, galactose and fructose uptake (Figure 3), but not of other hexoses (Figure 3 and Supplementary Figure S2).

In conclusion, CsSWEET proteins from clades I, II, and IV transport hexoses, such as fructose, galactose, mannose, and particularly glucose. CsSWEET proteins from clade III transport sucrose, rather than hexoses, which is similar to results from A. thaliana and rice (Figure 3 ).

\section{Spatiotemporal Expression Analysis of the CsSWEET Genes}

In order to determine the spatiotemporal expression pattern of each CsSWEET gene, the transcriptional profile in different cucumber organs was analyzed using qRT-PCR. Samples of fruit at the marketable fruit maturation stage 9 DAA (days after anthesis) (Hu et al., 2011) and other organs, including a source organ (mature leaf), sink organs (root, male flower, female flower, fruit, and young leaf) and a transporting organ (stem), were isolated. The eight analyzed CsSWEET genes from the four clades were expressed in almost all organs (Figure 4I), and at very high levels in male and female flowers, especially in the case of CsSWEET7b, $-10,-12 c$, and $-17 c$, where the expression was approximately 10-100 times greater than in other organs (Figure 4). But beyond that, the genes from different clades showed distinct expression patterns. In clade I, CsSWEET1 and CsSWEET2 were highly expressed in mature leaves, with CsSWEET1 also showing high expression in roots and CsSWEET2 in 9 DAA fruit, while they both showed low expression in the other organs (Figures 4A,B). In clade II, CsSWEET5a showed high expression in mature leaves, and low expression in roots and stems. CsSWEET7b was mainly expressed in roots, with little expression in stems, source leaves and fruit (Figures 4C,D). In clade III, CsSWEET10 was specifically expressed in fruit, while CsSWEET12c was expressed only in the stems and flowers (Figures 4E,F). In clade IV, CsSWEET17a was expressed in almost all organs, but with a high level in young leaves and mature leaves, while CsSWEET17c was highly expressed in stems besides flowers, and less in mature leaves and fruits (Figures 4G,H). 

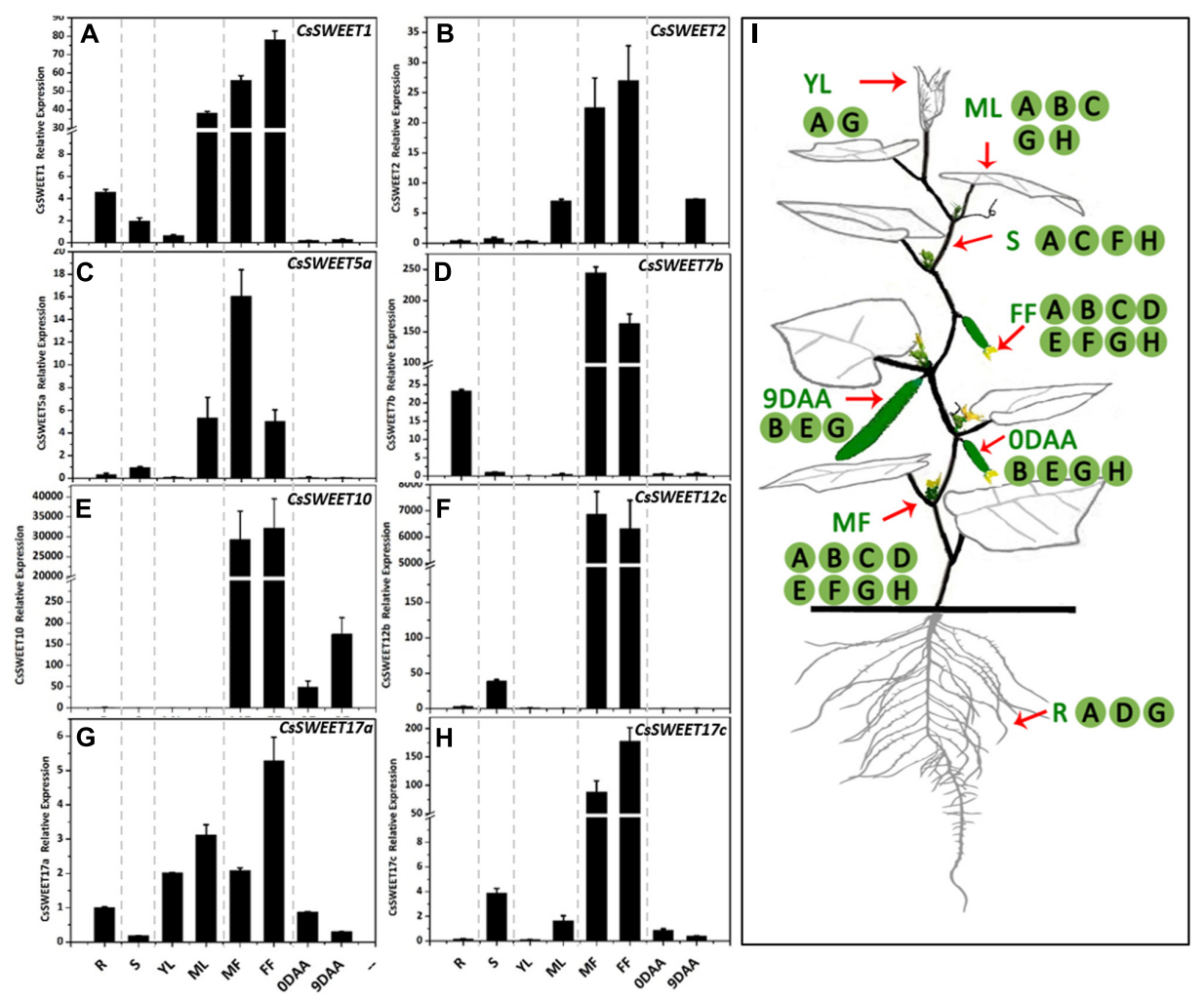

FIGURE 4 | The expression patterns of eight CsSWEET genes. (A-H) Relative expression of CsSWEETs. R, root; S, stem; YL, young leaf; ML, mature leaf; MF, male flower; FF, female flower; ODAA, ovary/fruit on the day of anthesis, 9DAA, fruit on the ninth day after anthesis. (I) Schematic model of the areas of CsSWEET gene expression in cucumber. Letters in (I) match the expression pattern in (A-H). Error bars represent the SE for three technical replicates of three biological replicates.

\section{Subcellular Localization of CsSWEET Proteins}

Previous studies have reported that different AtSWEET proteins have different subcellular localizations (Chen et al., 2015a; Eom et al., 2015), which in turn is suggestive of distinct functions. Here, the subcellular localization of the cucumber CsSWEET proteins was determined by expressing CsSWEETsGFP fusion proteins in A. thaliana protoplasts. We found that CsSWEET1-GFP from clade I, CsSWEET7b-GFP from clade II and CsSWEET12c-GFP from clade III were targeted to the plasma membrane, while CsSWEET17a-GFP from clade IV localized to the tonoplast (Figure 5). These results were also verified in chloroplast-free onion (Allium cepa) epidermal cells (Supplementary Figure S1), suggesting that proteins from clades I, II, and III are plasma membrane proteins that may be involved in transmembrane transportation between cells, while clade IV proteins are tonoplast transporters, and may be involved in transportation and accumulation inside cells and in balancing intracellular hexose homeostasis.

\section{DISCUSSION}

In the earlier work ( $\mathrm{Hu}$ et al., 2017), a genome-wide characterization of SWEET genes was conducted in cucumber
(Cucumis sativus L.) including a phylogenetic tree of the SWEET genes in cucumber, $A$. thaliana and rice, analysis of ciselements in promoter regions, chromosome distribution, gene structure and an analysis of transcript levels which provided a basic understanding of the CSSWEET genes. In their study, 17 CSSWEETs were identified which were not evenly distributed over the seven cucumber chromosomes. Besides that, cis-elements were identified in the promoter regions: nine types involved in phytohormone responses and eight types involved in stress responses (Hu et al., 2017). SWEETs have been reported as playing vital roles in embryo and seed development, phloem loading, nectary secretion, and other important life processes (Chen et al., 2012, 2015a; Lin et al., 2014; Sosso et al., 2015). These functions mostly depend on their subcellular localization and transport substrates. Based on the earlier work, phylogenetic analyses, yeast uptake assays, qRT-PCR and GFP fusion protein localization were used to elucidate the function of SWEET genes in cucumber in this study.

\section{CsSWEET Genes Fall into Four Phylogenetic Clades}

In this study, 17 putative cucumber SWEET genes were identified, classified into four clades (I-IV), and named according to their homologs in A. thaliana and earlier work (Hu et al., 2017). The overall sequence identity of all cucumber SWEET proteins is $42 \%$, 


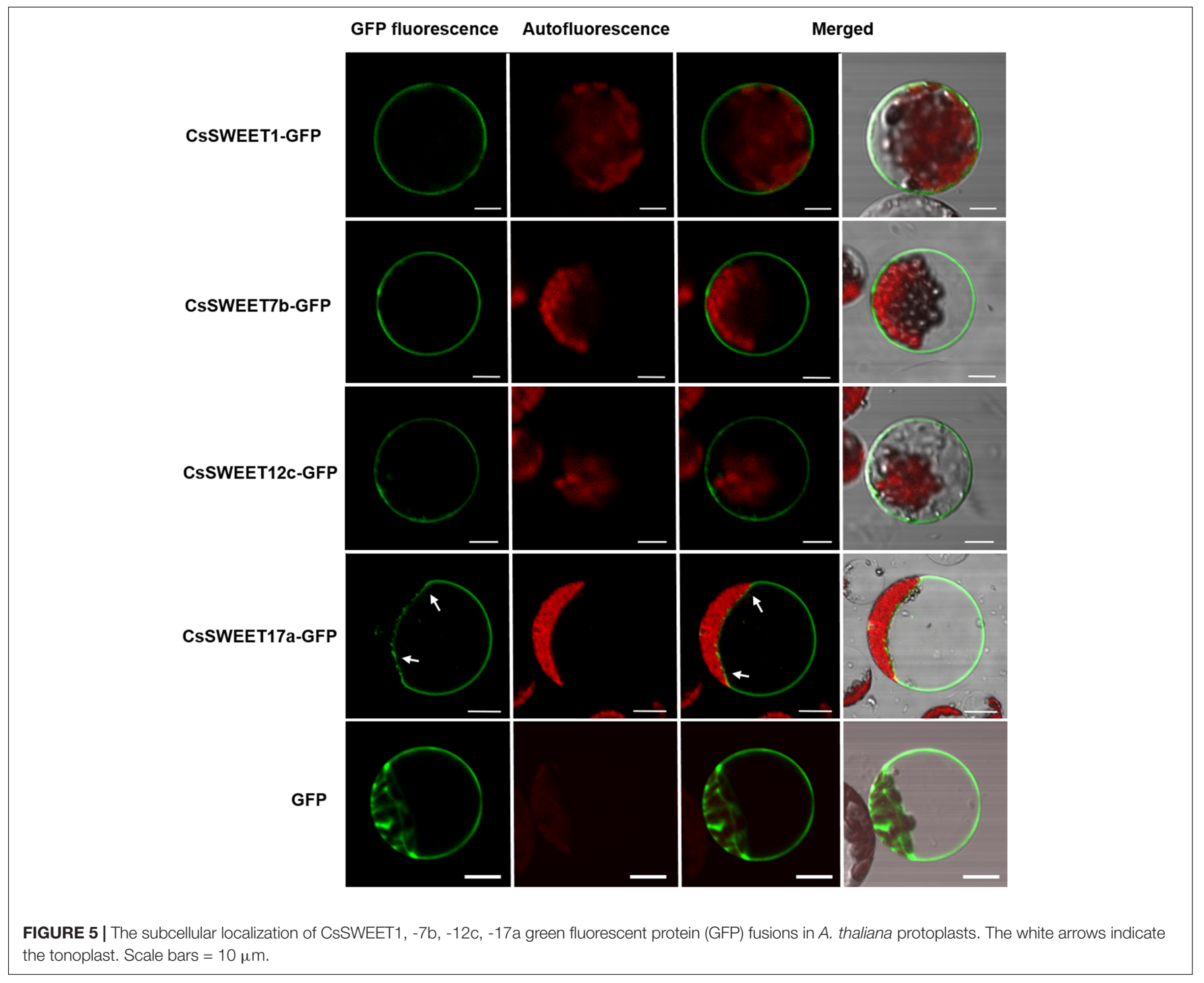

while the equivalent identity for the A. thaliana SWEET proteins is $43 \%$, and the degree of identity among all SWEET proteins shown in the phylogenetic tree was $33.03 \%$ (Figure 1). We identified six cucumber SWEET genes in clade III, five genes in clade II, and three genes in each of clades I and IV. This is similar to the numbers in A. thaliana and other plant species. In clade III, A. thaliana has 7 genes, tomato (Solanum lycopersicum) has 13, potato (Solanum tuberosum) has 15 , and Medicago truncatula has 10 (Feng et al., 2015; Manck-Götzenberger and Requena, 2016). This relatively high number may be related to the important role of sucrose in long distance transport. In contrast there are only three clade IV genes in cucumber and potato, one in M. truncatula and rice, and two in A. thaliana and tomato.

\section{Substrate Specificity of CsSWEET Proteins}

We determined that CsSWEET proteins from clades I and II could mediate uptake of hexose, especially glucose, in the yeast EBY.VW4000 mutant, which has also been reported for their homologs from $A$. thaliana (Chen et al., 2010, 2015a). In our study, we detected some differences between the uptake preferences of the different CsSWEET proteins: CsSWEET2 allowed fructose uptake, CsSWEET5a allowed the uptake of fructose and glucose, while CsSWEET7b conferred the uptake of glucose, and to a degree mannose, as well as galactose (Figure 3). In other species, less research has been done on substrate specificity. ZmSWEET4c and OsSWEET4 function as glucose and fructose transporters, VvSWEET4 encodes a glucose transporter and OsSWEET5 encodes a galactose transporter in yeast (Guo et al., 2014; Zhou et al., 2014; Sosso et al., 2015). Clade III was reported to be a sucrose-specific clade in A. thaliana (Chen et al., 2012), CsSWEET10 and CsSWEET 12c, also belong to clade III and mediated the uptake of sucrose, but not hexoses in the EBY.VW4000 mutant. In clade IV, the A. thaliana protein AtSWEET17 was found to be a fructose-specific transporter (Guo et al., 2014), while CsSWEET17a rescued glucose uptake and CsSWEET17c rescued glucose, galactose, and fructose uptake in the yeast mutant. 
We therefore conclude that there may exist some differences in the functions of the SWEET proteins among species, and that the SWEET proteins generally have a broader substrate not only uptake glucose but other hexoses. Recently, it has been found that SWEETs have the functions of transporting plant hormone, such as gibberellin (Kanno et al., 2016).

\section{The Probable Biological Functions of the CsSWEET Proteins}

Hu et al. (2017) predicted the following subcellular localizations of CsSWEETs by WoLF PSORT (a localization prediction tool); CsSWEET1 in the chloroplast/vacuole, CsSWEET12c and CsSWEET17a in the chloroplast. In our study, using GFP fusions, we discovered that CsSWEET1, CsSWEET7b, and CsSWEET12c are plasma membrane proteins, while CsSWEET17a is a vacuole membrane protein (Figure 5). These results, obtained through experimental tests differ from those of $\mathrm{Hu}$ et al. (2017) predicted by bioinformatics software/tools.

In this study, all of the eight tested CsSWEET genes were most highly expressed in male and female flowers, which are strong carbon sinks. SWEET proteins have been reported as having roles in nectar secretion and also tapetum and pollen formation (Guan et al., 2008; Sun et al., 2013; Lin et al., 2014), and in flowers, a lack of sugar availability may lead to flower abortion (Lebon et al., 2008; Chong et al., 2014). CsSWEET1 and CsSWEET7b showed high expression in roots and flowers, and may be involved in the uptake and release of carbohydrates in these organs, or alternatively function in an interaction between roots and soil microorganisms. They may also play important roles in sugar partitioning during flower and root growth development (Figures 4, 5). This is similar to AtSWEET4, which is a hexose transporter that mediates sugar transport to axial sinks (Liu et al., 2016). ZmSWEET4c and OsSWEET4 also appeared to be responsible for transferring hexoses across the BETL to sustain development of the large starch-storing endosperm of cereal grains and contribute to sink strength (Sosso et al., 2015).

Most studies of SWEET proteins in sink organs have focused on flowers, seeds or roots (Guan et al., 2008; Chardon et al., 2013; Sun et al., 2013; Guo et al., 2014; Chen et al., 2015b). The roles in fleshy sink organs, such as fruits, is a new and important area of research. In cucumber fruits, phloem unloading follows an extensive apoplastic pathway and needs transporter participation (Hu et al., 2011). In this study, CsSWEET2 and CsSWEET10 showed high levels of expression in fruit and their substrates were found to be hexoses and sucrose, respectively. Accordingly, sucrose may be released into the phloem parenchymal cells by CsSWEET10 transmembrane transport and glucose and fructose derived from sucrose degradation may be released into the phloem parenchymal cells by CsSWEET2. Their high expression levels in fruit is consistent with a possible role in apoplastic sugar unloading and fruit development.

In contrast, CsSWEET17a was found to be a vacuolar transporter (Figure 5) and showed 47.1\% similarity with CsSWEET17c. They are both in clade IV and show 41.55 and $51.8 \%$ similarity with AtSWEET17, respectively, which is known to be a vacuolar transporter (Chardon et al., 2013; Guo et al., 2014). They were expressed in almost all the sampled organs as they are tonoplast proteins. We speculate that these genes, from clade IV, play a role in facilitating hexose transport across the tonoplast and in balancing intracellular hexose homeostasis.

\section{CONCLUSION}

Based on their spatiotemporal expression patterns and subcellular localization, we propose that the CsSWEET proteins from clades I, II, and III may participate in the distribution of carbohydrate between organs through the phloem, because these corresponding proteins are localized in the plasma membrane. CsSWEET proteins from clade IV may be involved in hexose transport and accumulation inside cells and in balancing intracellular hexose homeostasis as they are targeted to the tonoplast. In future studies, the biological functions of the individual CsSWEET proteins will be investigated through histochemical localization and reverse genetics approaches.

\section{AUTHOR CONTRIBUTIONS}

ZZ, XS, and YL designed the research; YL, SF, and SM performed the research; YL and SF analyzed the data; YL, ZZ, and XS wrote the paper.

\section{FUNDING}

This work was supported by the National Natural Science Foundation of China (31471876), the Ministry of Agriculture of China (project no. 2013ZX08009), Beijing Innovation Consortium of Agriculture Research System (BAIC01-2017), and 111 project (B17043).

\section{ACKNOWLEDGMENTS}

We thank PlantScribe (www.plantscribe.com) for careful editing of this manuscript. We thank Dr. Shubin Sun (Nanjing Agricultural University) for the gift of the yeast strain EBY.VW4000, Dr. Dapeng Zhang (Tsinghua University) for the gift of the yeast strain SUSY7/ura and pDR196 vector. We are grateful to Chenggang Xiang (Chinese Academy of Agricultural Sciences) for help with analysis of the distribution of CsSWEETs on chromosomes.

\section{SUPPLEMENTARY MATERIAL}

The Supplementary Material for this article can be found online at: https://www.frontiersin.org/articles/10.3389/fpls.2017.01855/ full\#supplementary-material 


\section{REFERENCES}

Antony, G., Zhou, J., Huang, S., Li, T., Liu, B., White, F., et al. (2010). Rice xa13 recessive resistance to bacterial blight is defeated by induction of the disease susceptibility gene Os-11N3. Plant Cell 22, 3864-3876. doi: 10.1105/tpc.110. 078964

Chardon, F., Bedu, M., Calenge, F., Klemens, P. A. W., Spinner, L., Clement, G., et al. (2013). Leaf fructose content is controlled by the vacuolar transporter SWEET17 in Arabidopsis. Curr. Biol. 23, 697-702. doi: 10.1016/j.cub.2013. 03.021

Chen, L., Hou, B., Lalonde, S., Takanaga, H., Hartung, M. L., Qu, X., et al. (2010). Sugar transporters for intercellular exchange and nutrition of pathogens. Nature 468, 527-532. doi: 10.1038/nature09606

Chen, L. Q. (2014). SWEET sugar transporters for phloem transport and pathogen nutrition. New Phytol. 201, 1150-1155. doi: 10.1111/nph.12445

Chen, L. Q., Cheung, L. S., Feng, L., Tanner, W., and Frommer, W. B. (2015a). Transport of Sugars. Annu. Rev. Biochem. 84, 865-894. doi: 10.1146/annurevbiochem-060614-033904

Chen, L. Q., Lin, I. W., Qu, X., Sosso, D., McFarlane, H. E., Londoño, A., et al. (2015b). A cascade of sequentially expressed sucrose transporters in the seed coat and endosperm provides nutrition for the Arabidopsis embryo. Plant Cell 27, 607-619. doi: 10.1105/tpc.114.134585

Chen, L. Q., Qu, X. Q., Hou, B. H., Sosso, D., Osorio, S., Fernie, A. R., et al. (2012). Sucrose efflux mediated by SWEET proteins as a key step for phloem transport. Science 335, 207-211. doi: 10.1126/science.1213351

Cheng, J., Wang, Z., Yao, F., Gao, L., Ma, S., Sui, X., et al. (2015). Downregulating CsHT1, a cucumber pollen-specific hexose transporter, inhibits pollen germination, tube growth, and seed development. Plant Physiol. 168, 635-647. doi: 10.1104/pp.15.00290

Chong, J., Piron, M. C., Meyer, S., Merdinoglu, D., Bertsch, C., and Mestre, P. (2014). The SWEET family of sugar transporters in grapevine: VvSWEET4 is involved in the interaction with Botrytis cinerea. J. Exp. Bot. 65, 6589-6601. doi: $10.1093 / \mathrm{jxb} / \mathrm{eru} 375$

Engel, M. L. (2005). Green sperm. Identification of male gamete promoters in Arabidopsis. Plant Physiol. 138, 2124-2133. doi: 10.1104/pp.104.054213

Eom, J., Chen, L., Sosso, D., Julius, B. T., Lin, I. W., Qu, X., et al. (2015). SWEETs, transporters for intracellular and intercellular sugar translocation. Curr. Opin. Plant Biol. 25, 53-62. doi: 10.1016/j.pbi.2015.04.005

Fan, R. C., and Zhang, D. P. (2009). Apple sucrose transporter SUT1 and sorbitol transporter SOT6 interact with cytochrome b5 to regulate their affinity for substrate sugars. Plant Physiol. 150, 1880-1901. doi: 10.1104/pp.109.141374

Feng, C., Han, J., Han, X., and Jiang, J. (2015). Genome-wide identification, phylogeny, and expression analysis of the SWEET gene family in tomato. Gene 573, 261-272. doi: 10.1016/j.gene.2015.07.055

Guan, Y. F., Huang, X. Y., Zhu, J., Gao, J. F., Zhang, H. X., and Yang, Z. N. (2008). RUPTURED POLLEN GRAIN1, a member of the MtN3/saliva gene family, is crucial for exine pattern formation and cell integrity of microspores in Arabidopsis. Plant Physiol. 147, 852-863. doi: 10.1104/pp.108.118026

Guo, W. J., Nagy, R., Chen, H. Y., Pfrunder, S., Yu, Y. C., Santelia, D., et al. (2014). SWEET17, a facilitative transporter, mediates fructose transport across the tonoplast of Arabidopsis roots and leaves. Plant Physiol. 164, 777-789. doi: 10.1104/pp.113.232751

Hu, L., Sun, H., Li, R., Zhang, L., Wang, S., Sui, X., et al. (2011). Phloem unloading follows an extensive apoplasmic pathway in cucumber (Cucumis sativus L.) fruit from anthesis to marketable maturing stage. Plant Cell Environ. 34, 1835-1848. doi: 10.1111/j.1365-3040.2011.02380.x

Hu, L., Zhang, F., Song, S., Tang, X., Xu, H., Liu, G., et al. (2017). Genomewide identification, characterization, and expression analysis of the SWEET gene family in cucumber. J. Integr. Agric. 16, 60345-60347. doi: 10.1016/S20953119(16)61501-0

Huang, S. W., Li, R. Q., Zhang, Z. H., Li, L., Gu, X. F., Wei, F., et al. (2009). The genome of the cucumber, Cucumis sativus L. Nat. Genet. 41, 1275-1281. doi: 10.1038/ng. 475

Kanno, Y., Oikawa, T., Chiba, Y., Ishimaru, Y., Shimizu, T., Sano, N., et al. (2016). AtSWEET13 and AtSWEET14 regulate gibberellin-mediated physiological processes. Nat. Commun. 7:13245. doi: 10.1038/ncomms13245
Lebon, G., Wojnarowiez, G., Holzapfel, B., Fontaine, F., Vaillantgaveau, N., and Clément, C. (2008). Sugars and flowering in the grapevine (Vitis vinifera L.). J. Exp. Bot. 59, 2565-2578. doi: 10.1093/jxb/ern135

Lin, I. W., Sosso, D., Chen, L., Gase, K., Kim, S., Kessler, D., et al. (2014). Nectar secretion requires sucrose phosphate synthases and the sugar transporter SWEET9. Nature 508, 546-549. doi: 10.1038/nature13082

Liu, Q., Yuan, M., Zhou, Y., Li, X., Xiao, J., and Wang, S. (2011). A paralog of the $\mathrm{MtN}$ /saliva family recessively confers race-specific resistance to Xanthomonas oryzae in rice. Plant Cell Environ. 34, 1958-1969. doi: 10.1111/j.1365-3040. 2011.02391.x

Liu, X., Zhang, Y., Yang, C., Tian, Z., and Li, J. (2016). AtSWEET4, a hexose facilitator, mediates sugar transport to axial sinks and affects plant development. Sci. Rep. 6:24563. doi: 10.1038/srep24563

Ma, N., Xue, J., Li, Y., Liu, X., Dai, F., Jia, W., et al. (2008). Rh-PIP2;1, a rose aquaporin gene, is involved in ethylene-regulated petal expansion. Plant Physiol. 148, 894-907. doi: 10.1104/pp.108.120154

Manck-Götzenberger, J., and Requena, N. (2016). Arbuscular mycorrhiza symbiosis induces a major transcriptional reprogramming of the potato SWEET sugar transporter family. Front. Plant Sci. 7:487. doi: 10.3389/fpls.2016. 00487

Mizuno, H., Kasuga, S., and Kawahigashi, H. (2016). The sorghum SWEET gene family: stem sucrose accumulation as revealed through transcriptome profiling. Biotechnol. Biofuels 9, 127. doi: 10.1186/s13068-016-0546-6

Riesmeier, J. W., Willmitzer, L., and Frommer, W. B. (1992). Isolation and characterization of a sucrose carrier cDNA from spinach by functional expression in yeast. EMBO J. 11, 4705-4713.

Seo, P. J., Park, J., Kang, S. K., Kim, S., and Park, C. (2011). An Arabidopsis senescence-associated protein SAG29 regulates cell viability under high salinity. Planta 233, 189-200. doi: 10.1007/s00425-010-1293-8

Sosso, D., Luo, D., Li, Q., Sasse, J., Yang, J., Gendrot, G., et al. (2015). Seed filling in domesticated maize and rice depends on SWEET-mediated hexose transport. Nat. Genet. 47, 1489-1493. doi: 10.1038/ng.3422

Sun, M., Huang, X., Yang, J., Guan, Y., and Yang, Z. (2013). Arabidopsis RPG1 is important for primexine deposition and functions redundantly with RPG2 for plant fertility at the late reproductive stage. Plant Reprod. 26, 83-91. doi: 10.1007/s00497-012-0208-1

Wieczorke, R., Krampe, S., Weierstall, T., Freidel, K., Hollenberg, C. P., and Boles, E. (1999). Concurrent knock-out of at least 20 transporter genes is required to block uptake of hexoses in Saccharomyces cerevisiae. FEBS Lett. 464, 123-128. doi: 10.1016/S0014-5793(99)01698-1

Wittich, P. E. (2000). NEC1, a novel gene, highly expressed in nectary tissue of Petunia hybrida. Plant J. 24, 725-734. doi: 10.1046/j.1365-313x.2000. 00926.x

Xuan, Y. H., Hu, Y. B., Chen, L. Q., Sosso, D., Ducat, D. C., Hou, B. H., et al. (2013). Functional role of oligomerization for bacterial and plant SWEET sugar transporter family. Proc. Natl. Acad. Sci. U.S.A. 110, E3685-E3694. doi: 10.1073/ pnas. 1311244110

Yuan, M., Chu, Z., Li, X., Xu, C., and Wang, S. (2009). Pathogen-induced expressional loss of function is the key factor in race-specific bacterial resistance conferred by a recessive R gene xa13 in rice. Plant Cell Physiol. 50, 947-955. doi: 10.1093/pcp/pcp046

Zhou, Y., Liu, L., Huang, W., Yuan, M., Zhou, F., Li, X., et al. (2014). Overexpression of OsSWEET5 in rice causes growth retardation and precocious senescence. PLOS ONE 9:e94210. doi: 10.1371/journal.pone.009 4210

Conflict of Interest Statement: The authors declare that the research was conducted in the absence of any commercial or financial relationships that could be construed as a potential conflict of interest.

Copyright (๑) 2017 Li, Feng, Ma, Sui and Zhang. This is an open-access article distributed under the terms of the Creative Commons Attribution License (CC BY). The use, distribution or reproduction in other forums is permitted, provided the original author(s) or licensor are credited and that the original publication in this journal is cited, in accordance with accepted academic practice. No use, distribution or reproduction is permitted which does not comply with these terms. 